\title{
Bilinear and quadratic Hamiltonians in two-mode cavity quantum electrodynamics
}

\author{
F. O. Prado ${ }^{1}$, N. G. de Almeida ${ }^{2}$, M. H. Y. Moussa ${ }^{1}$ and C. J. Villas-Bôas ${ }^{1}$ \\ ${ }^{1}$ Universidade Federal de São Carlos, São Carlos, Brazil and \\ ${ }^{2}$ Universidade Católica de Goiás, Goiânia, Brazil
}

\begin{abstract}
In this work we show how to engineer bilinear and quadratic Hamiltonians in cavity quantum electrodynamics (QED) through the interaction of a single driven two-level atom with cavity modes. The validity of the engineered Hamiltonians is numerically analyzed even considering the effects of both dissipative mechanisms, the cavity field and the atom. The present scheme can be used, in both optical and microwave regimes, for quantum state preparation, the implementation of quantum logical operations, and fundamental tests of quantum theory.
\end{abstract}




\section{INTRODUCTION}

Frequency-conversion mechanisms, such as optical parametric and four-wave mixing processes, have acted as a basic resource in the investigation of fundamental quantum phenomena over the last few decades. Largely employed to produce squeezed and polarizationentangled photon states to test sub-Poissonian statistics 1] and Bell's inequalities [2], such processes have deepened our understanding of radiation [1] and its interaction with matter 3]. Apart from applications in fundamental physics, it has been conjectured that frequency conversions can improve the signal-to-noise ratio in optical communication 4] and be used to measure gravitational waves through squeezed fields [5]. Recently, they have also been required within quantum information theory for the implementation of a nondeterministic controlled-NOT operation [6].

Against this backdrop of the general usefulness of frequency conversions in the runningwave domain, several recent studies have been devoted to mapping these mechanisms into two-mode cavity quantum electrodynamics (QED) [7, 8, 9, 10]. Parametric up- and downconversions (PUC and PDC) were accomplished through the dispersive interactions of a single three-level atom simultaneously with a classical driving field and a two-mode cavity. The PDC (PUC) process follows from the ladder (lambda) configuration of atomic levels, in which the ground $|g\rangle$ and excited $|e\rangle$ states are coupled through an auxiliary intermediate (more-excited) level $|i\rangle$. The cavity modes $\omega_{a}$ and $\omega_{b}$ are tuned to the vicinity of the dipoleallowed transitions $|g\rangle \leftrightarrow|i\rangle$ and $|e\rangle \leftrightarrow|i\rangle$. The desired interaction between the modes $\omega_{a}$ and $\omega_{b}, \hbar\left(\xi a b+\xi^{*} a^{\dagger} b^{\dagger}\right)$ for PDC or $\hbar\left(\xi a b^{\dagger}+\xi^{*} a^{\dagger} b\right)$ for PUC, is accomplished by driving the dipole-forbidden atomic transition $|g\rangle \leftrightarrow|e\rangle$ out of resonance with a classical field. For the

degenerate PDC process, where $\omega_{a}=\omega_{b}$, the well-known interaction $\hbar\left[\xi(a)^{2}+\xi^{*}\left(a^{\dagger}\right)^{2}\right]$ was first achieved in [7], and this may be used to squeeze an arbitrary state previously prepared in the cavity; i.e., to perform the squeezing operation $S|\Psi\rangle$ in cavity QED ( $S$ being the squeeze operator). These achievements enhance prospects of quantum information manipulation and of fundamental tests of quantum theory in cavity QED. In fact, the engineered bilinear Hamiltonians can be used to generate one-mode mesoscopic squeezed superpositions, twomode entanglements, and two-mode squeezed vacuum states (such the original EPR state).

Motivated by these accomplishments [7, 8, 9, 10], and simultaneously attempting to generalize and simplify these protocols, in the present letter we consider only a two-level 
Rydberg atom in order to generate, in two-mode cavity QED, bilinear and quadratic Hamiltonians similar to those describing PUC and PDC. We also demonstrate how to generate, in one-mode cavity QED, the anti-Jaynes-Cummings (AJC) Hamiltonian 11] and a mixture of the Jaynes-Cummings (JC) and the AJC Hamiltonians. We stress that all the previous schemes presented in literature to generate nonlinear Hamiltonians consider the interaction between a cavity mode and an atom with at least three atomic levels. The present protocol, overcomes the difficulty of driving the dipole-forbidden atomic transition $|g\rangle \leftrightarrow|e\rangle$, which sometimes requires a significant strength, as in the strong amplification regime defined in Ref. [9]. More importantly, with a two-level atom, long-lived circular Rydberg states might be employable reducing the noise coming from the finite lifetimes of the atomic levels. (We stress that, to achieve PUC and PDC with a three-level atomic configuration, at least one level cannot be a long-lived circular Rydberg state.) Our results are derived from variations of the Hamiltonian $H=H_{0}+V(t)$ (with $\hbar=1$ ), with

$$
\begin{aligned}
H_{0} & =\omega_{a} a^{\dagger} a+\omega_{b} b^{\dagger} b+\omega_{0}\left(\sigma_{e e}-\sigma_{g g}\right) / 2, \\
V(t) & =\left[\lambda_{a} a \sigma_{e g}+\lambda_{b} b \sigma_{e g}+\left(\Omega_{1} \mathrm{e}^{-i \omega_{1} t}+\Omega_{2} \mathrm{e}^{-i \omega_{2} t}\right) \sigma_{e g}+\text { h.c. }\right],
\end{aligned}
$$

where the atomic ground $(g)$ and excited $(e)$ states, with transition frequency $\omega_{0}$, are coupled non-resonantly through the cavity modes of frequencies $\omega_{a}$ and $\omega_{b}$, with coupling constants $\lambda_{a}$ and $\lambda_{b}$, and detunings $\delta_{a}=\omega_{0}-\omega_{a}$ and $\delta_{b}=\omega_{0}-\omega_{b}$. The dipole-allowed transition $|g\rangle$ $\leftrightarrow|e\rangle$ is also excited by two driving classical fields of frequencies $\omega_{1}$ and $\omega_{2}$, with coupling constants $\Omega_{1}=\left|\Omega_{1}\right| \mathrm{e}^{i \varphi_{1}}$ and $\Omega_{2}=\left|\Omega_{2}\right| \mathrm{e}^{i \varphi_{2}}$, and detunings $\delta_{1}=\omega_{0}-\omega_{1}$ and $\delta_{2}=\omega_{0}-\omega_{2}$. In the interaction picture, the transformed Hamiltonian is given by

$$
V_{I}(t)=\lambda_{a} \mathrm{e}^{i \delta_{a} t} a \sigma_{e g}+\lambda_{b} \mathrm{e}^{i \delta_{b} t} b \sigma_{e g}+\left(\Omega_{1} \mathrm{e}^{i \delta_{1} t}+\Omega_{2} \mathrm{e}^{i \delta_{2} t}\right) \sigma_{e g}+\text { h.c.. }
$$

\section{BILINEAR AND QUADRATIC HAMILTONIANS}

A single classical amplification field $\left(\omega_{1}\right)$ is required to accomplish these interactions. After writing the Hamiltonian $V_{I}(t)$ in this laser framework and defining a new basis for the atomic states $\left\{| \pm\rangle \simeq\left(\mathrm{e}^{i \varphi_{1}}|e\rangle \pm|g\rangle\right) / \sqrt{2}\right\}[11]$, under the assumption that $\delta_{1} \ll\left|\Omega_{1}\right|,\left|\delta_{a}\right|$, $\left|\delta_{b}\right|$, we proceed to the transformation $\mathcal{U}=\exp \left[-i\left|\Omega_{1}\right|\left(\sigma_{++}-\sigma_{--}\right) t\right]$, which prepares the Hamiltonian

$$
\mathcal{V}(t)=\left(\widetilde{\lambda}_{a} \mathrm{e}^{i\left(\delta_{a}-\delta_{1}\right) t} a+\widetilde{\lambda}_{b} \mathrm{e}^{i\left(\delta_{b}-\delta_{1}\right) t} b\right)\left(\sigma_{++}-\sigma_{--}-\mathrm{e}^{2 i \Omega_{1} t} \sigma_{+-}+\mathrm{e}^{-2 i \Omega_{1} t} \sigma_{-+}\right) / 2+\text { h.c. }
$$


with $\widetilde{\lambda}_{\alpha}=\lambda_{\alpha} \mathrm{e}^{-i \varphi_{1}}(\alpha=a, b)$, for a subsequent perturbation approximation. We finally obtain, up to the second order, the effective Hamiltonian [12]

$$
\mathcal{H}=-i \mathcal{V}(t) \int \mathcal{V}\left(t^{\prime}\right) d t^{\prime}
$$

which will be analysed under three regimes of the classical amplification field: the weak $\left(\left|\delta_{a}\right| \sim\left|\delta_{b}\right| \gg\left|\Omega_{1}\right| \gtrsim\left|\widetilde{\lambda}_{a}\right| \sim\left|\tilde{\lambda}_{b}\right|\right)$, the intermediate $\left(\left|\Omega_{1}\right| \sim\left|\delta_{a}\right| \sim\left|\delta_{b}\right| \gg\left|\tilde{\lambda}_{a}\right| \sim\left|\widetilde{\lambda}_{b}\right|\right)$ and the strong $\left(\left|\Omega_{1}\right| \gg\left|\delta_{a}\right| \sim\left|\delta_{b}\right| \gg\left|\widetilde{\lambda}_{a}\right| \sim\left|\widetilde{\lambda}_{b}\right|\right)$ amplification regimes.

It is important to note from Eq. (3) that once the relation $\left|2 \Omega_{1} \pm\left(\delta_{\alpha}-\delta_{1}\right)\right| \gg\left|\widetilde{\lambda}_{\alpha}\right|$ is satisfied for all the amplification regimes, there will be no transition between the atomic dressed states $|+\rangle$ and $|-\rangle$ (even that there will be transitions between the bare states $|g\rangle$ and $|e\rangle)$.

\section{A. The Hamiltonian $(a b+$ h.c. $)$}

From the above analysis, this interaction is achieved considering the energy diagram pictorially sketched in Fig. 1(a), where $\delta_{a}=-\delta_{b}=\delta>0$. From Eq. (41), we obtain the effective Hamiltonian $\mathcal{H}(t)=\mathcal{H}_{0}+\mathcal{H}_{\text {int }}(t)$, with

$$
\begin{aligned}
\mathcal{H}_{0} & =\frac{\left|\Omega_{1}\right|}{4\left|\Omega_{1}\right|^{2}-\delta^{2}}\left(\left|\widetilde{\lambda}_{a}\right|^{2} a^{\dagger} a+\left|\widetilde{\lambda}_{b}\right|^{2} b^{\dagger} b\right)\left(\sigma_{++}-\sigma_{--}\right) \\
& +\frac{2}{\delta} \sum_{\ell=+,-}\left(\left|\widetilde{\lambda}_{a}\right|^{2} \frac{\delta+\ell\left|\Omega_{1}\right|}{\delta+2 \ell\left|\Omega_{1}\right|}-\left|\widetilde{\lambda}_{b}\right|^{2} \frac{\delta-\ell\left|\Omega_{1}\right|}{\delta-2 \ell\left|\Omega_{1}\right|}\right) \sigma_{\ell \ell} \\
\mathcal{H}_{\text {int }}(t) & =\left(\frac{\widetilde{\lambda}_{a} \widetilde{\lambda}_{b}\left|\Omega_{1}\right|}{\delta^{2}-4\left|\Omega_{1}\right|^{2}} \mathrm{e}^{-2 i \delta_{1} t} a b+\text { h.c }\right)\left(\sigma_{++}-\sigma_{--}\right) .
\end{aligned}
$$

Preparing the atomic state $| \pm\rangle$, we obtain through the unitary transformation $\mathcal{H}_{ \pm}=$ $U^{\dagger} \mathcal{H}(t) U-\mathcal{H}_{0}$, with $U=\mathrm{e}^{-i \mathcal{H}_{0} t}$, the engineered interaction

$$
\mathcal{H}_{ \pm}=\left(\Lambda_{ \pm} a b+\text { h.c. }\right)
$$

where the coupling parameters in the weak $(W)$, intermediate $(I)$ and strong $(S)$ amplification regimes become $\Lambda_{ \pm W}= \pm \widetilde{\lambda}_{a} \widetilde{\lambda}_{b}\left|\Omega_{1}\right| / \delta^{2}, \Lambda_{ \pm I}= \pm \widetilde{\lambda}_{a} \widetilde{\lambda}_{b}\left|\Omega_{1}\right| /\left(\delta^{2}-4\left|\Omega_{1}\right|^{2}\right)$, and $\Lambda_{ \pm S}=$ $\mp \tilde{\lambda}_{a} \widetilde{\lambda}_{b} / 4\left|\Omega_{1}\right|$, after adjusting the detuning $\delta_{1}$ such that $\delta_{1 W}= \pm\left(\left|\widetilde{\lambda}_{a}\right|^{2}+\left|\widetilde{\lambda}_{b}\right|^{2}\right)\left|\Omega_{1}\right| / \delta^{2}$, $\delta_{1 I}= \pm\left(\left|\widetilde{\lambda}_{a}\right|^{2}+\left|\widetilde{\lambda}_{b}\right|^{2}\right)\left|\Omega_{1}\right| /\left(4\left|\Omega_{1}\right|^{2}-\delta^{2}\right)$, and $\delta_{1 S}= \pm\left(\left|\widetilde{\lambda}_{a}\right|^{2}+\left|\widetilde{\lambda}_{b}\right|^{2}\right) / 4\left|\Omega_{1}\right|$, respec- 
tively. (For the intermediate amplification regime care must be take to avoid the equality $\left|\Omega_{1}\right|=|\delta|$.) We note that the strength of the coupling parameters obey the relation $\Lambda_{I} \gtrsim \Lambda_{W} \gtrsim \Lambda_{S}$

\section{B. The Hamiltonian (ab $b^{\dagger}+$ h.c.)}

The energy diagram leading to this interaction is sketched in Fig. 1(b), where $\delta_{a} \sim \delta_{b}$. The Effective Hamiltonian becomes

$$
\begin{aligned}
\mathcal{H}_{0} & =\left(\frac{\left|\Omega_{1}\right|\left|\widetilde{\lambda}_{a}\right|^{2}}{4\left|\Omega_{1}\right|^{2}-\delta_{a}^{2}} a^{\dagger} a+\frac{\left|\Omega_{1}\right|\left|\widetilde{\lambda}_{b}\right|^{2}}{4\left|\Omega_{1}\right|^{2}-\delta_{b}^{2}} b^{\dagger} b\right)\left(\sigma_{++}-\sigma_{--}\right) \\
& +\frac{1}{2} \sum_{\ell=+,-}\left(\left|\widetilde{\lambda}_{a}\right|^{2} \frac{\delta_{a}+\ell\left|\Omega_{1}\right|}{\delta_{a}\left(\delta_{a}+2 \ell\left|\Omega_{1}\right|\right)}+\left|\widetilde{\lambda}_{b}\right|^{2} \frac{\delta_{b}+\ell\left|\Omega_{1}\right|}{\delta_{b}\left(\delta_{b}+2 \ell\left|\Omega_{1}\right|\right)}\right) \sigma_{\ell \ell}, \\
\mathcal{H}_{i n t}(t) & =\frac{1}{2} \widetilde{\lambda}_{a} \widetilde{\lambda}_{b}^{*} \mathrm{e}^{i\left(\delta_{a}-\delta_{b}\right) t} a b^{\dagger} \sum_{\ell=+,-}\left(\frac{\delta_{b}+\ell\left|\Omega_{1}\right|}{\delta_{b}\left(\delta_{b}+2 \ell\left|\Omega_{1}\right|\right)}-\frac{\delta_{a}-\ell\left|\Omega_{1}\right|}{\delta_{a}\left(\delta_{a}-2 \ell\left|\Omega_{1}\right|\right)}\right) \sigma_{\ell \ell}+\text { h.c.. }
\end{aligned}
$$

Again, preparing the atomic state $| \pm\rangle$ we obtain, through the same steps leading to the interaction $(a b+$ h.c. $)$, the effective Hamiltonian

$$
\mathcal{H}_{ \pm}=\left(\Sigma_{ \pm} a b^{\dagger} \mathrm{e}^{i \Phi_{ \pm} t}+\text { h.c. }\right),
$$

where the phase $\Phi_{ \pm}= \pm\left|\Omega_{1}\right|\left(\frac{\left|\tilde{\lambda}_{b}\right|^{2}}{4\left|\Omega_{1}\right|^{2}-\delta_{b}^{2}}-\frac{\left|\tilde{\lambda}_{a}\right|^{2}}{4\left|\Omega_{1}\right|^{2}-\delta_{a}^{2}}\right)+\delta_{a}-\delta_{b}$ can be made null only in the intermediate regime where the term in brackets, multiplied by $\left|\Omega_{1}\right|$, can be made of the order of the detuning $\delta_{a}-\delta_{b}$. The coupling parameters $\Sigma_{ \pm W}=\widetilde{\lambda}_{a} \widetilde{\lambda}_{b}^{*}\left(\delta_{a}-\delta_{b}\right) / 2 \delta_{a} \delta_{b}$, $\Sigma_{ \pm I}= \pm \widetilde{\lambda}_{a} \widetilde{\lambda}_{b}^{*}\left|\Omega_{1}\right| /\left(4\left|\Omega_{1}\right|^{2}-\delta_{a} \delta_{b}\right)$, and $\Sigma_{ \pm S}= \pm \widetilde{\lambda}_{a} \widetilde{\lambda}_{b}^{*} / 4\left|\Omega_{1}\right|$, follows without any need to adjust the detuning $\delta_{1}$, since the condition $\delta_{1} \ll\left|\Omega_{1}\right|,\left|\delta_{a}\right|,\left|\delta_{b}\right|$ is satisfied. Again, the strength of the coupling parameters obey the relation $\Sigma_{I} \gtrsim \Sigma_{W} \gtrsim \Sigma_{S}$. The time-dependent Hamiltonian (8) can be treated through the invariants introduced by Lewis and Riesenfeld [13], as discussed in Ref. [14, 15]. Otherwise, we may consider identical modes $\omega_{a}=\omega_{b}$ (so that $\delta_{a}=\delta_{b}$ ) of two identical cavities disposed along perpendicular transversal axes and sharing the same two-level atom.

\section{Applications}

As mentioned above, the engineered bilinear Hamiltonians can be used for quantum state preparation in cavity QED. In Refs. [8, 15] the interaction (6) was employed in a protocol 
for the preparation of the original Einstein-Podolsky-Rosen (EPR) entanglement expanded in the Fock representation. This interaction was also required to engineer the even and odd EPR states defined in Ref. [9]. The advantage of the protocol in [9] over that in [8] is the use of a intense classical amplification field, where the strength of the bilinear interactions between the cavity modes are considerably increased, at least by one order of magnitude, compared to the strength in [8]. Consequently, the atom-field interaction time required to obtain high-fidelity states can be considerably shorter, making the dissipative effects negligible.

The present scheme, in turn, has the advantage over those in Refs. [8, 9], in that a two-level atom is able to generate both interactions, (66) and (8), with coupling strengths comparable to those obtained in [9]. Therefore, apart from the benefit of a shorter interaction time, due to the strength of the coupling parameters $\Lambda$ and $\Sigma$, here we get an additional advantage employing circular long-lived Rydberg states. The same facilities apply to the squeezing Hamiltonian engineered below. We finally mention that, following the reasoning in Ref. [6], our engineered bilinear interactions can be considered to manipulate quantum information in cavity QED.

\section{THE SQUEEZING HAMILTONIAN}

To obtain the parametric amplification Hamiltonian, we consider, as indicated in Fig. 2, the atomic transition coupled to a single cavity mode $\left(\omega_{a}\right)$, as well as two classical amplification fields, with $\delta_{1}=0$ and $\delta_{2}<0$, under the condition $\left|\Omega_{1}\right|=-\delta_{2} / 2 \gg\left|\widetilde{\lambda}_{a}\right|,\left|\Omega_{2}\right|,\left|\delta_{a}\right|$. Starting from the interaction picture we obtain, after the unitary transformation $U_{1}=$ $\exp \left[-i\left(\Omega_{1} \sigma_{e g}+\Omega_{1}^{*} \sigma_{g e}\right) t\right]$ and within the rotating wave approximation, the interaction

$$
\widetilde{V}_{1}(t)=\widetilde{\lambda}_{a} a \mathrm{e}^{i \delta_{a} t}\left(\sigma_{++}-\sigma_{--}\right) / 2-\widetilde{\Omega}_{2} \sigma_{+-}+\text {h.c. }
$$

where $\widetilde{\Omega}_{2}=\Omega_{2} \mathrm{e}^{-i \varphi_{1}} / 2$. Through the new basis $\left\{|\uparrow\rangle=\left(\mathrm{e}^{i\left(\varphi_{2}-\varphi_{1}\right)}|+\rangle \pm|-\rangle\right) / \sqrt{2}\right\}$ it is straightforward to verify that, after another transformation $U_{2}=\exp \left[i\left(\widetilde{\Omega}_{2} \sigma_{+-}+\right.\right.$h.c. $\left.) t\right]$, the interaction (91) becomes

$$
\widetilde{V}_{2}(t)=\left(\widetilde{\lambda}_{a} a \mathrm{e}^{i \delta_{a} t}+\widetilde{\lambda}_{a}^{*} a^{\dagger} \mathrm{e}^{-i \delta_{a} t}\right)\left(\sigma_{\uparrow \downarrow} \mathrm{e}^{-i\left|\Omega_{2}\right| t}+\sigma_{\downarrow \uparrow} \mathrm{e}^{i\left|\Omega_{2}\right| t}\right),
$$

which is suitable for the derivation, under the assumption that $\left|\delta_{a}\right| \ll\left|\Omega_{2}\right|$, of the effective Hamiltonian $\mathcal{H}=-i \widetilde{V}_{2}(t) \int \widetilde{V}_{2}\left(t^{\prime}\right) d t^{\prime}$. Finally, for the initial atomic state $\left|\begin{array}{l}\uparrow \\ \downarrow\end{array}\right\rangle$ and adjusting 
$\delta_{a\left(\begin{array}{l}\uparrow \\ \downarrow\end{array}\right)}=\mp 2\left|\widetilde{\lambda}_{a}\right|^{2} /\left|\Omega_{2}\right|$, we obtain the engineered parametric amplification Hamiltonian

$$
\mathcal{H}_{(\uparrow)}=\mp \chi\left(\mathrm{e}^{-2 i \varphi_{1}} a^{2}+\text { h.c. }\right)
$$

where $\chi=\left|\widetilde{\lambda}_{\alpha}^{2} / 4 \Omega_{2}\right|$, which allows the squeezing of any desired prepared cavity-field state. We note that the squeezing direction in phase space is controlled through the phase factor $\mathrm{e}^{-i \varphi_{1}}$ derived from a classical amplification field. With $\varphi_{1}=\varphi_{2}=0$ we recover the atomic bases $\{|g\rangle,|e\rangle\}$ in that $|\uparrow\rangle=|e\rangle$ and $|\downarrow\rangle=|g\rangle$. Differently from the protocols with threelevel atoms [8, 9, 10, 15], where the squeezing interaction is engineered through degenerate atomic transitions, here a second amplification field on a single atomic transition is required to achieve the two-photon process.

\section{A. Applications}

In the running-wave domain, the squeezed states revealed the intrinsic quantum nature of light, together with direct evidence for an atom undergoing a quantum jump [3]. The engineered interaction (11) exposes a myriad of possible applications in cavity QED, ranging from the preparation of a set of squeezed states [16] to the possibility of revealing the statistical properties of the electromagnetic field through its controlled interaction with atoms. Beyond these applications, a particular squeezed superposition state (SSS) can be prepared when adjusting $\delta_{a}=\varphi_{1}=\varphi_{2}=0$, such that the Hamiltonian governing the evolution of the atom-field state reads

$$
\mathcal{H}=-\chi\left[2 a^{\dagger} a+a^{2}+\left(a^{\dagger}\right)^{2}\right]\left(\sigma_{e e}-\sigma_{g g}\right)
$$

Starting from the initial state $(|e\rangle+|g\rangle)|\alpha\rangle / \sqrt{2},|\alpha\rangle$ being a coherent state injected into the cavity, the generated SSS is $\left(|e\rangle U_{e}+|g\rangle U_{g}\right)|\alpha\rangle / \sqrt{2}$, where $U_{\ell}$ stands for the evolution operator associated with Hamiltonian $H_{\ell}=\langle\ell|\mathcal{H}| \ell\rangle$, with $\ell=e, g$. It has been shown in Ref. 14] that the decoherence time of this particular SSS - where both states composing the superposition exhibt the same squeezing direction - could be delayed to around the relaxation time of the cavity field. This remarkable result requires the engineering of absolute zero reservoirs composed by oscillators squeezed in a direction perpendicular to that of the superposition state. The reason behind this phenomenon is quite palpable: the injection of 
noise from the reservoir into the superposition state decreases as the degree of squeezing, of both the reservoir and the superposition states, increases. Therefore, the present scheme is a crucial step towards the accomplishment of this specific program for protecting a quantum state. (We stress that the engineering of an ideally squeezed reservoir for a cavity mode, a task only partially achieved in Ref. 17], has also been accomplished by our group [18].)

\section{THE AJC HAMILTONIAN}

To engineer the AJC model in cavity QED we start from the interaction $\widetilde{V}_{2}(t)$ that leads to the squeezing operator. Considering $\varphi_{1}=\varphi_{2}=0$ and $\delta_{a}=-\left|\Omega_{2}\right|$, we obtain, within

the rotating-wave approximation following from the condition $\left|\Omega_{2}\right| \gg\left|\widetilde{\lambda}_{a}\right|^{2}$, the desired interaction

$$
\mathcal{H}_{A J C}=\widetilde{\lambda}_{a} a \sigma_{g e}+\text { h.c. }
$$

which has already been engineered in cavity QED with two-level [11] or three-level atoms [19]. While in Ref. [11] the AJC interaction is achieved, with a single classical field, by adjusting the detuning between the cavity mode and the atomic transition, in the present scheme we adjust the detuning between the classical field $\omega_{2}$ and the atomic transition. It is also possible to obtain an alternation between the JC and the AJC model assuming the same parameters that lead to the AJC model $\left(\varphi_{1}=\varphi_{2}=0,\left|\delta_{a}\right|=-\left|\Omega_{2}\right|\right.$, and $\left.\left|\Omega_{2}\right| \gg\left|\widetilde{\lambda}_{a}\right|\right)$. To this end, two synchronized pulsed fields must be introduced, leading to $\omega_{2}(t)=\omega_{0}-\delta_{2}(t)$, with $\delta_{2}(t)=\left|\delta_{2}\right|\left[\Theta_{1}(t)-\Theta_{2}(t)\right]$. Given $\Theta_{\ell}(t)=\sum_{n} \Theta\left[t-\left(2 n+\delta_{\ell 2}\right) \tau\right] \Theta\left[\left(2 n+1+\delta_{\ell 2}\right) \tau-t\right], \tau$ being the duration of each pulse and $n$ an integer, the engineered Hamiltonian reads

$$
\mathcal{H}=\Theta_{1}(t)\left(\widetilde{\lambda}_{a} a \sigma_{g e}+\text { h.c. }\right)+\Theta_{2}(t)\left(\widetilde{\lambda}_{a} a \sigma_{e g}+\text { h.c. }\right) \text {. }
$$

\section{DISCUSSION AND CONCLUSION}

Next, addressing some sensitive points in the present engineering scheme, we turn our attention to the effective squeezing interaction in Eq. (11), to demonstrate that it follows with good agreement from the full Hamiltonian in Eq. (11). In Fig. 3, starting with the cavity field in the vacuum state and the atom in the ground state, we plot the variance of the cavity-field squeezed quadrature, $(\Delta X)^{2}$, against the squeezing factor $r=2 \chi t$. Although the present scheme can be applied to both optical and microwave regimes, in Fig. 3 we have 
used typical parameters from cavity QED experiments in the microwave regime, which, in units of $\lambda_{a}=3 \times 10^{5} \mathrm{~s}^{-1}$, are approximately given by: $\omega_{0}=10^{5}, \Omega_{1}=4 \times 10^{2}, \Omega_{2}=20$, $\delta_{2}=8 \times 10^{2}$. The solid line corresponds to the variance computed either analytically or numerically from the effective squeezing interaction (111) (the exceedingly small difference between the two curves being around $0.3 \%$ for $r=1$ ). The dashed line corresponds to the variance computed numerically from the full Hamiltonian in Eq. (11). Fig. 3 reveals a good agreement between the solid and dashed lines, for $r$ ranging from zero to unity, with the degree of squeezing being $86.4 \%$ and $85.6 \%$, respectively. Evidently, these degrees of squeezing can even be enhanced considering a sample of $N$ noninteracting atoms instead of a single one, as considered in [10].

We also computed the effects of both dissipative mechanisms, the cavity field and the atom, on the degree of squeezing achieved. The dotted line traces the numerical computation of the variance of the squeezed quadrature based on the master equation

$$
\dot{\rho}=-i[H, \rho]+\mathcal{L}_{\text {field }} \rho+\mathcal{L}_{\text {atom }} \rho
$$

with $H$ given by Eq. (11). As usual for a reservoir at absolute zero, the Liouville operators read: $\mathcal{L}_{\text {field }} \bullet=\left(\Gamma_{f} / 2\right)\left(2 a \bullet a^{\dagger}-a^{\dagger} a \bullet-\bullet a^{\dagger} a\right)$ and $\mathcal{L}_{\text {atom }} \bullet=$ $\left(\Gamma_{a} / 2\right)\left(2 \sigma_{-} \bullet \sigma_{+}-\sigma_{+} \sigma_{-} \bullet-\bullet \sigma_{+} \sigma_{-}\right)$. We again assumed, in units of $\lambda_{a}$, typical values for high-finesse cavities and circular-Rydberg states, around $\Gamma_{f}=3 \times 10^{-3}$ and $\Gamma_{a}=10^{-4}$. From Fig. 3 we observe that the degree of squeezing under the dissipative effects, falls to $80.5 \%$, which is still a remarkable result.

In this work we have presented protocols to build bilinear and quadratic Hamiltonians in cavity QED, employing a single two-level atom plus classical amplification fields. The simplicity and generality of these schemes make them suitable for the implementation of quantum logical operations [6], quantum state preparation [9], and fundamental tests of quantum theory [20]. The validity of the approximations leading to our effective Hamiltonians has been confirmed with numerical calculations, even under the effect of dissipative mechanisms. As well as deepening our understanding of atom-field interaction in cavity QED, our protocols may also open the way to advances in correlated areas such as trappedion and nanomechanical oscillators.

\section{Acknowledgments}

We wish to express thanks for the support from FAPESP (Projeto Temático Número 
00/15084-5), CNPq (Instituto do Milênio de Informação Quântica), and CAPES, Brazilian agencies, and to Tim Roberts for a critical reading of the manuscript.

[1] D. Stoler, Phys. Rev. Lett. 33, 1397 (1974).

[2] P. G. Kwiat, K. Mattle, H. Weinfurter, A. Zeilinger, A. V. Sergienko and Y. Shih, Phys. Rev. Lett. 75, 4337 (1995).

[3] H. J. Kimble, M. Dagenais, and L. Mandel, Phys. Rev. Lett. 39, 691 (1977).

[4] H. P. Yuen and J. H. Shapiro, IEEE Trans. Inf. Theory 24, 657 (1978); D. J. Wineland, J. J. Bollinger, W. M. Itano, and D. J. Heinzen, Phys. Rev. A 50, 67 (1994).

[5] J. N. Hollenhorst, Phys. Rev. D 19, 1669 (1979); C. M. Caves, K. S. Thorne, R. W. P. Drever, V. D. Sandberg, and M. Zimmermann, Rev. Mod. Phys. 52, 341 (1980).

[6] E. Knill, R. Laflamme, G. J. Milburn, Nature 409, 46 (2001); T. B. Pittman, B. C. Jacobs, and J. D. Franson, Phys. Rev. Lett. 88, 257902 (2002).

[7] C. J. Villas-Boas, N. G. de Almeida, R. M. Serra, and M. H. Y. Moussa, Phys. Rev. A 68, 061801(R) (2003).

[8] R. M. Serra, C. J. Villas-Boas, N. G. de Almeida, and M. H. Y. Moussa, Phys. Rev. A 71, $045802(2005)$.

[9] C. J. Villas-Boas and M. H. Y.Moussa, European Physical Journal D 32,147 (2005).

[10] R. Guzmán, J. C. Retamal, E. Solano,and N. Zagury, Phys. Rev. Lett. 96, 010502 (2006); quant-ph/0407218.

[11] E. Solano, G. S. Agarwal, and H. Walther, Phys. Rev. Lett. 90, 027903 (2003). See also S. B. Zheng, Phys. Rev. A 66, 060303(R) (2002).

[12] D. F. V. James, Fortschr. Phys. 48, 823 (2000).

[13] H. R. Lewis and W. B. Riesenfeld, J. Math. Phys. 10, 1458 (1969).

[14] C. J. Villas-Boas, F. R. de Paula, R. M. Serra, and M. H. Y. Moussa, Phys. Rev. A 68, 053808 (2003); J. Opt. B: Quantum Semiclass. Opt. 5, 391 (2003).

[15] C. J. Villas-Boas, N. G. de Almeida, R. M. Serra, and M. H. Y. Moussa, Phys. Rev. A 68, 061801(R) (2003).

[16] N. G. de Almeida, R. M. Serra, C. J. Villas-Boas, and M. H. Y. Moussa, Phys. Rev. A 69, $035802(2004)$. 
[17] P. Tombesi and D. Vitali, Phys. Rev. A 50, 4253 (1994).

[18] F. O. Prado, M. H. Y. Moussa, and C. J. Villas-Bôas, to appear elsewhere.

[19] M. F. Santos, E. Solano, and R. L. de Matos Filho, Phys. Rev. Lett. 87, 093601 (2001).

[20] A. Rauschenbeutel, P. Bertet, S. Osnaghi, G. Nogues, M. Brune, J. M. Raimond, and S. Haroche, Phys. Rev. A 64, 050301(R) (2001); B. T. H. Varcoe, S. Brattke, M. Weidinger, and H. Walther, Nature 403, 743 (2000).

Fig. 1. Energy diagrams underlying the bilinear Hamiltonians (a) $a b+$ h.c. and (b) $a b^{\dagger}+$ h.c.

Fig. 2. Energy diagram of the scheme used to obtain the squeezing interaction.

Fig. 3. Variance of the cavity-field squeezed quadrature $(\Delta X)^{2}$ plotted against the squeezing factor $r$ for the effective squeezing interaction in Eq. (11) (solid line), the full Hamiltonian in Eq. (11) (dashed line), and the full Hamiltonian under cavity-field and atomic decay (dotted line). 
Fig.1(a)

Fig.1(b)

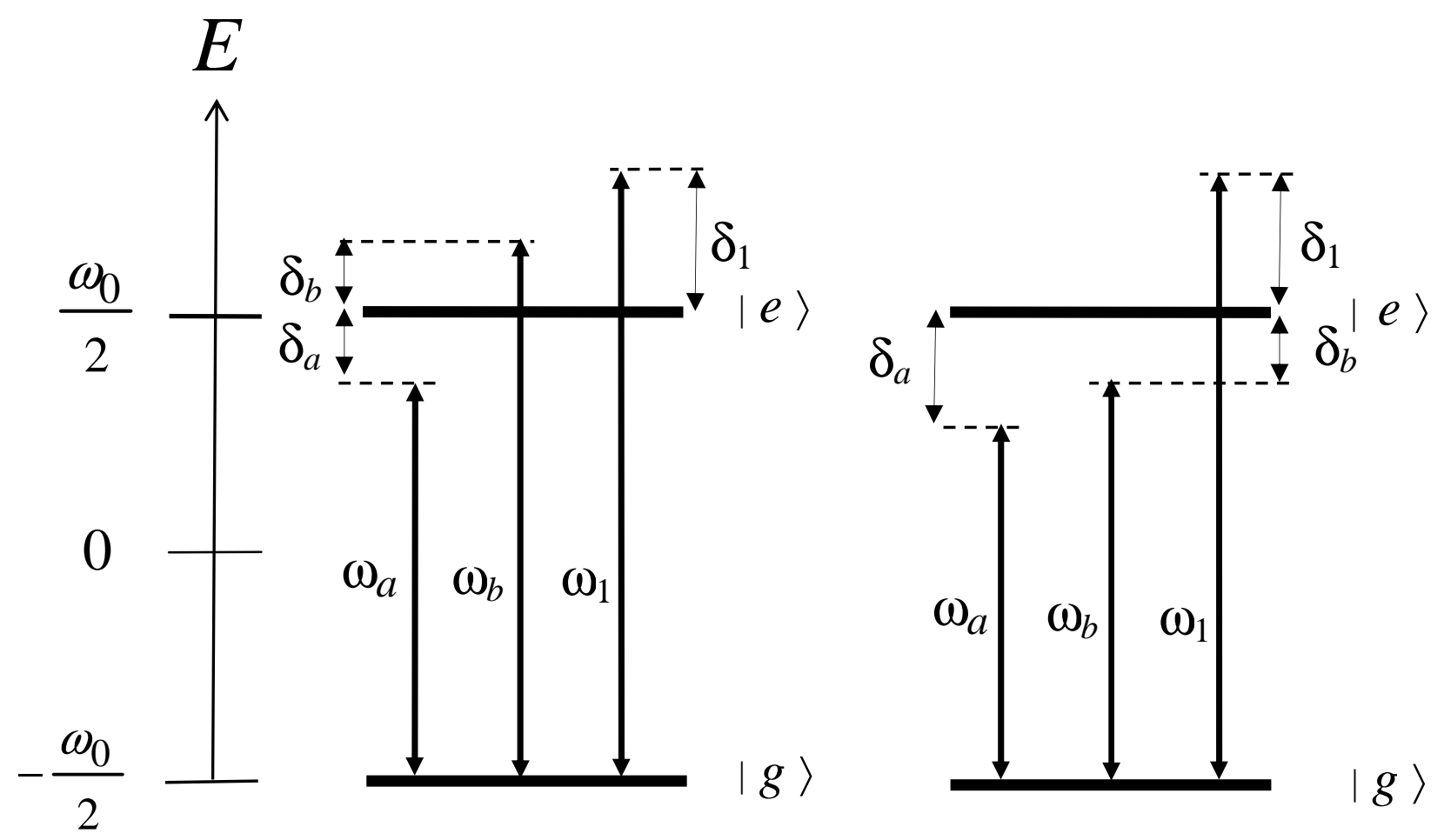


Fig. 2

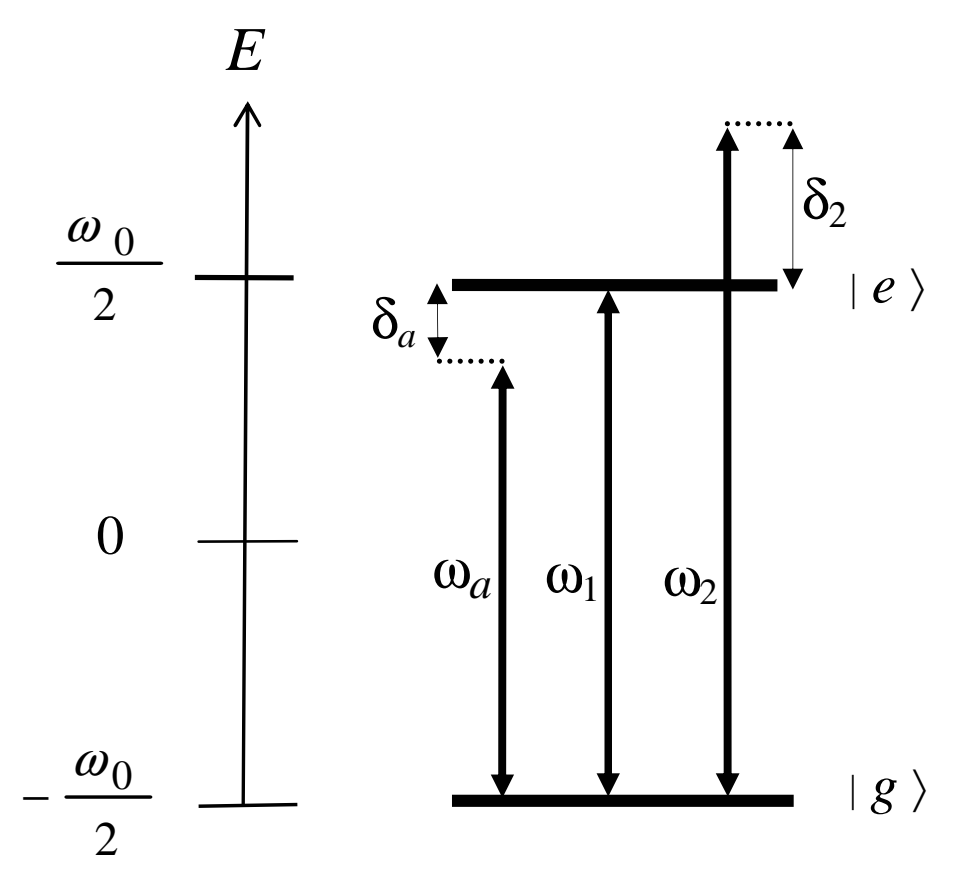


Fig. 3

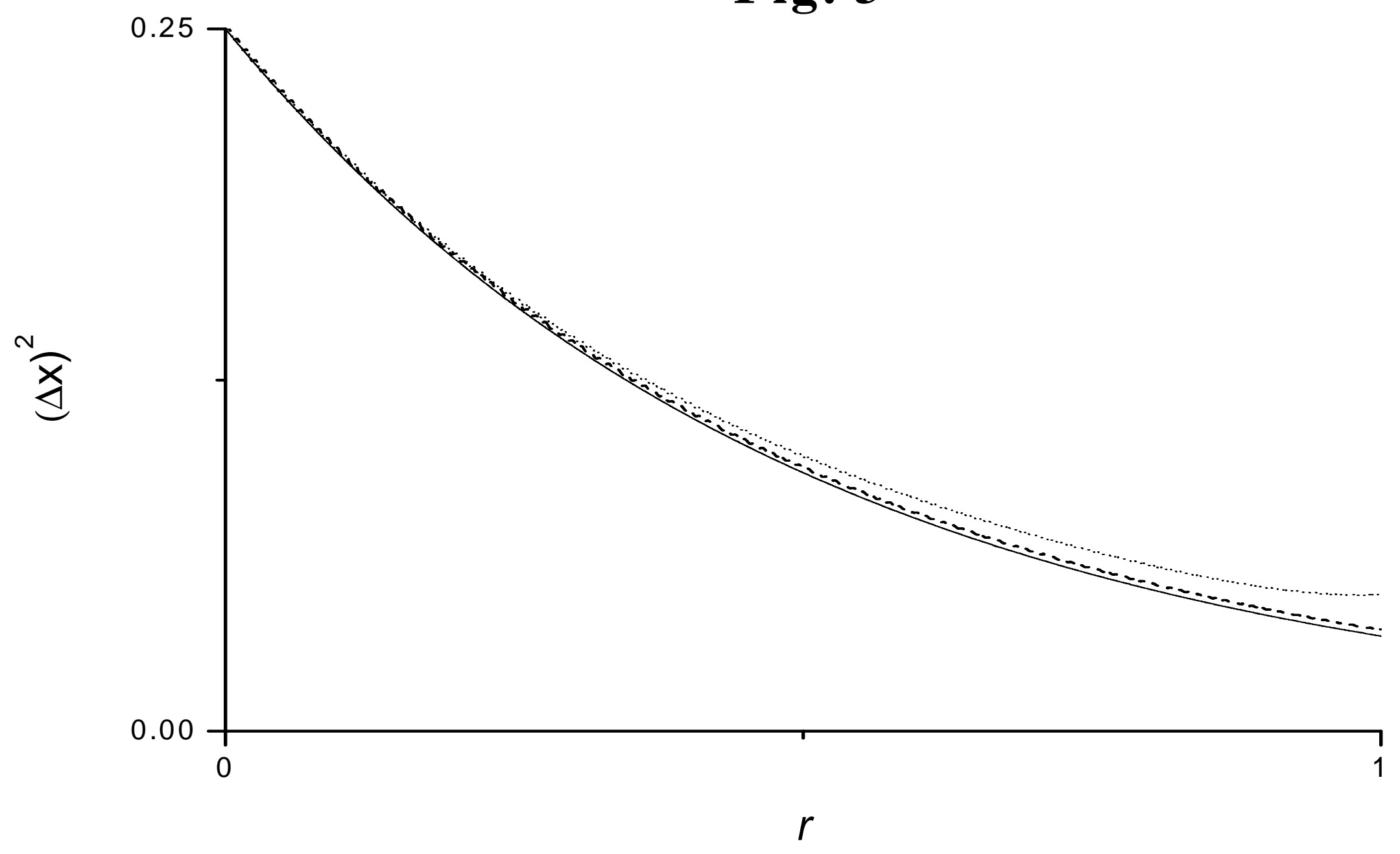

